\title{
Secure and Reliable Transmission with Cooperative Relays in Two-Hop Wireless Networks
}

\author{
Yulong Shen*§, Xiaohong Jiang ${ }^{\dagger}$, Jianfeng $\mathrm{Ma}^{*}$ and Weisong Shi ${ }^{\ddagger}$ \\ ${ }^{*}$ School of Computer Science and Technology, Xidian University, China \\ ${ }^{\dagger}$ School of Systems Information Science, Future University Hakodate, Japan \\ $\ddagger$ Department of Computer Science, Wayne State University, USA \\ §Email:ylshen@mail.xidian.edu.cn
}

\begin{abstract}
This work considers the secure and reliable information transmission in two-hop relay wireless networks without the information of both eavesdropper channels and locations. While the previous work on this problem mainly studied infinite networks and their asymptotic behavior and scaling law results, this papers focuses on a more practical network with finite number of system nodes and explores the corresponding exact results on the number of eavesdroppers the network can tolerant to ensure a desired secrecy and reliability. For achieving secure and reliable information transmission in a finite network, two transmission protocols are considered in this paper, one adopts an optimal but complex relay selection process with less load balance capacity while the other adopts a random but simple relay selection process with good load balance capacity. Theoretical analysis is further provided to determine the exact and maximum number of independent and also uniformly distributed eavesdroppers one network can tolerate to satisfy a specified requirement in terms of the maximum secrecy outage probability and maximum transmission outage probability allowed.
\end{abstract}

\section{INTRODUCTION}

Two-hop ad hoc wireless networks, where each packet travels at most two hops (source-relay-destination) to reach its destination, has been a class of basic and important networking scenarios [1]. Actually, the analysis of basic two-hop relay networks serves as the foundation for performance study of general multi-hop networks. Due to the promising applications of ad hoc wireless networks in many important scenarios (like battlefield networks, emergency networks, disaster recovery networks), the consideration of secrecy (and also reliability) in such networks is of great importance for ensuring the high confidentiality requirements of these applications. This paper focuses on the issue of secure and reliable information transmission in the basic two-hop ad hoc wireless networks.

Traditionally, the information security is provided by adopting the cryptography approach, where a plain message is encrypted through a cryptographic algorithm that is hard to break (decrypt) in practice by any adversary without the key. While the cryptography is acceptable for general applications with standard security requirement, it may not be sufficient for applications with a requirement of strong form of security (like military networks and emergency networks). This is because that the cryptographic approach can hardly achieve everlasting secrecy, since the adversary can record the transmitted messages and try any way to break them [2]. That is why there is an increasing interest in applying signaling scheme in physical layer to provide a strong form of security, where a degraded signal at an eavesdropper is always ensured such that the original data can be hardly recovered regardless of how the signal is processed at the eavesdropper. We consider applying physical layer method to guarantee secure and reliable information transmission in the two-hop wireless networks.

By now, a lot of research efforts have been dedicated to providing security through physical layer methods. A power control scheme is proposed in [3] to ensure that an eavesdropper can never reach its desired signal-to-noiseplus-interference ratio (SINR). However, such scheme is not effective when the eavesdropper has a better channel than the receiver. The technique of artificial noise generation has also been widely explored to jam the eavesdroppers and provide secure transmission in the relay communications [4][5] [6][7]. Recently, the cooperative jamming through node cooperation has been demonstrated to be efficient in ensuring physical layer security [8][9][10]. It is notable that these schemes generally reply on the knowledge of eavesdropper channels and locations to jam eavesdroppers. In practice, however, it is difficult to gain such information, specifically in untrusted network environment. To address this constraint, a cooperative protocol based on artificial noise generation and multi-user diversity has been proposed recently in [11] to achieve secure transmission in two-hop wireless networks without the knowledge of eavesdropper channels and locations. In particular, the asymptotic behavior of such cooperative protocol in a network has been reported there to illustrate how the number of eavesdroppers the network can tolerate scales as the number of system nodes there tends to infinite.

This paper focuses on applying the relay cooperation scheme to achieve secure and reliable information transmission in a more practical finite two-hop wireless network without the knowledge of both eavesdropper channels and locations. The main contributions of this paper as follows:

1) For achieving secure and reliable information transmission in a more practical two-hop wireless network with finite number of system nodes, we consider the application of the cooperative protocol proposed in [11] with an optimal and complex relay selection process but less load balance capacity, and also propose to use a new cooperative protocol with a simple and random relay selection process 


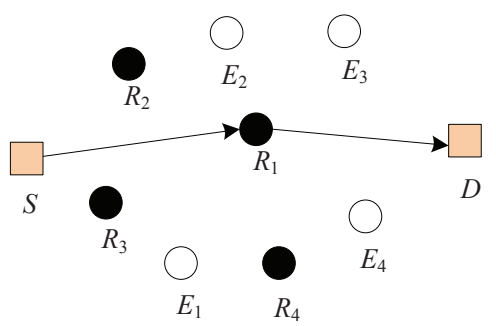

Fig. 1. System scenario: Source $S$ wishes to communicate securely with destination $D$ with the assistance of finite relays $R_{1}, R_{2}, \cdots, R_{n}$ ( $n=4$ in the figure) in the presence of passive eavesdroppers $E_{1}, E_{2}, \cdots, E_{m}(m=4$ in the figure). Cooperative relay scheme is used in the two-hop transmission. A assistant node is selected randomly as relay $\left(R_{1}\right.$ in the figure).

but good load balance capacity.

2) Rather than exploring the asymptotic behavior and scaling law results, this paper provides theoretic analysis of above both cooperative protocols to determine the corresponding exact results on the number of independent and also uniformly distributed eavesdroppers one network can tolerate to satisfy a specified requirement in terms of the maximum secrecy outage probability and maximum transmission outage probability allowed.

The remainder of the paper is organized as follows. Section II introduces the system models and two cooperative transmission protocols considered in this paper. Section III provides theoretical analysis and also related discussions of the two protocols, and Section IV concludes this paper.

\section{System Models And Transmission Protocols}

\section{A. Network Model}

As illustrated in Fig.1 that we consider a network scenario where a source node $S$ wishes to communicate securely with its destination node $D$ with the help of multiple relay nodes $R_{1}, R_{2}, \cdots, R_{n}$. In addition to these normal system nodes, there are also $m$ eavesdroppers $E_{1}, E_{2}, \cdots, E_{m}$ that are independent and also uniformly distributed in the network. Our goal here is to ensure the secure and reliable information transmission from source $S$ to destination $D$ under the condition that no real time information is available about both eavesdropper channels and locations.

\section{B. Transmission Model}

Consider the transmission from a transmitter $A$ to a receiver $B$, and denote by $x_{i}^{(A)}$ the $i^{t h}$ symbol transmitted by $A$ and denote by $y_{i}^{(B)}$ the $i^{\text {th }}$ signal received by $B$. We assume that all nodes transmit with the same power $E_{s}$, path loss between all pairs of nodes is equal and independent, and the frequencynonselective multi-path fading from $A$ to $B$ is a complex zeromean Gaussian random variable. Under the condition that all nodes in a group of nodes, $\mathcal{R}$, are generating noises, the $i^{t h}$ signal received at node $B$ from node $A$ is determined as:

$$
y_{i}^{(B)}=h_{A, B} \sqrt{E_{s}} x_{i}^{(A)}+\sum_{A_{i} \in \mathcal{R}} h_{A_{i}, B} \sqrt{E_{s}} x_{i}^{\left(A_{i}\right)}+n_{i}^{(B)},
$$

where the noise $\left\{n_{i}^{(B)}\right\}$ at receiver $B$ is assumed to be i.i.d complex Gaussian random variables with $E\left[\left|n_{i}^{(B)}\right|^{2}\right]=$ $N_{0}$, and $\left|h_{A, B}\right|^{2}$ is exponentially distributed with mean $E\left[\left|h_{A, B}\right|^{2}\right]$. Without loss of generality, we assume that $E\left[\left|h_{A, B}\right|^{2}\right]=1$. The SINR $C_{A, B}$ from $A$ to $B$ is then given

$$
C_{A, B}=\frac{E_{s}\left|h_{A, B}\right|^{2}}{\sum_{A_{i} \in \mathcal{R}} E_{s}\left|h_{A_{i}, B}\right|^{2}+N_{0} / 2}
$$

For a legitimate node and an eavesdropper, we use two separate SINR thresholds $\gamma_{R}$ and $\gamma_{E}$ to define the minimum SINR required to recover the transmitted messages for legitimate node and eavesdropper, respectively. Therefore, a system node (relay or destination) is able to decode a packet if and only if its SINR is greater than $\gamma_{R}$, while the transmitted message is secure if and only if the SINR at each eavesdropper is less than $\gamma_{E}$.

\section{Transmission Protocols}

We consider here two transmission protocols for secure and reliable information transmission in two-hop wireless networks. The first protocol (hereafter called Protocol 1) is the one proposed in [11], in which the optimal relay node with the best link condition to both source and destination is always selected for information relaying. Although this protocol is attractive in the sense that it provide very effective resistance against eavesdroppers, it suffers from several problems. The protocol 1 involves a complicated process of optimal relay selection, which is not very suitable for the distributed wireless networks, in particular when the number of possible relay nodes is huge. More importantly, since the channel state is relatively constant during a fixed time period, some relay nodes with good link conditions are always preferred for information relaying, resulting a severe load balance problem and a quick node energy depletion in energy-limited wireless environment.

Based on these observations, we propose to use a simple and random relay selection rather than the optimal relay selection to achieve a better load and energy consumption balance among possible relay nodes. By modifying the Protocol 1 to include the random relay selection process, the new transmission protocol (hereafter called Protocol 2) works as follows.

1) Relay selection: A relay node, indexed by $j^{*}$, is selected randomly from candidate relay nodes $R_{j}, j=1,2, \cdots, n$.

2) Channel measurement between the selected relay and the other relays: The selected relay $j^{*}$ broadcasts a pilot signal to allow each of other relays to measure the channel from $j^{*}$ to itself. Each of the other relays $R_{j}, j=1,2, \cdots, n, j \neq j^{*}$ then knows the corresponding value of $h_{R_{j}, R_{j^{*}}}$.

3) Channel measurement between destination $D$ and the other relays: The destination $D$ broadcasts a pilot signal to allow each of other relays to measure the channel from $D$ to 
itself. Each of the other relays $R_{j}, j=1,2, \cdots, n, j \neq j^{*}$ then knows the corresponding value of $h_{R_{j}, D}$.

4) Message transmission from source $S$ to the selected relay $R_{j^{*}}$ : The source $S$ transmits the messages to $R_{j^{*}}$. Concurrently, the relay nodes with indexes in $\mathcal{R}_{1}=$ $\left\{j \neq j^{*}:\left|h_{R_{j}, R_{j^{*}}}\right|^{2}<\tau\right\}$, transmit noise to generate sufficient interference at eavesdroppers.

5) Message transmission from the selected relay $R_{j^{*}}$ to destination $D$ : Similar to the Step 4, the relay $R_{j^{*}}$ transmits the messages to destination $D$. Concurrently, the relay nodes with indexes in $\mathcal{R}_{2}=\left\{j \neq j^{*}:\left|h_{R_{j}, D}\right|^{2}<\tau\right\}$, transmit noise to generate sufficient interference at eavesdroppers.

\section{TheORETICAL ANALYsis}

This section first defines the transmission outage and secrecy outage adopted in this paper to depict transmission reliability and transmission secrecy, and then provides theoretical analysis to determine the numbers of eavesdroppers a network can tolerate based on the Protocol 1 and Protocol 2, respectively.

\section{A. Transmission Outage and Secrecy Outage}

For a transmission from the source $S$ to destination $D$, we call transmission outage happens if $D$ can not decode the transmitted packet, i.e., $D$ received the packet with SINR less than the predefined threshold $\gamma_{R}$. The transmission outage probability, denoted as $P_{o u t}^{(T)}$, is then defined as the probability that transmission outage from $S$ to $D$ happens. For a predefined upper bound $\varepsilon_{t}$ on $P_{\text {out }}^{(T)}$, we call the communication between $S$ and $D$ is reliable if $P_{o u t}^{(T)} \leq \varepsilon_{t}$. Notice that for the transmissions from $S$ to the selected relay $R_{j^{*}}$ and from $R_{j^{*}}$ to $D$, the corresponding transmission outage can be defined in the similar way as that of from $S$ to $D$. We use $O_{S \rightarrow R_{j^{*}}}^{(T)}$ and $O_{R_{j^{*} \rightarrow D}}^{(T)}$ to denote the events that transmission outage from source $S$ to $R_{j^{*}}$ happens and transmission outage from relay $R_{j^{*}}$ to $D$ happens, respectively. Due to the link independence assumption, we have

$$
\begin{aligned}
P_{\text {out }}^{(T)}= & P\left(O_{S \rightarrow R_{j^{*}}}^{(T)} \cup O_{R_{j^{*} \rightarrow D}^{(T)}}^{(T)}\right) \\
= & P\left(O_{S \rightarrow R_{j^{*}}}^{(T)}\right)+P\left(O_{R_{j^{*} \rightarrow D} \rightarrow D}^{(T)}\right) \\
& -P\left(O_{S \rightarrow R_{j^{*}}}^{(T)}\right) \cdot P\left(O_{R_{j^{*} \rightarrow D}^{(T)}}\right)
\end{aligned}
$$

Regarding the secrecy outage, we call secrecy outage happens for a transmission from $S$ to $D$ if at least one eavesdropper can recover the transmitted packets during the process of this two-hop transmission, i.e., at least one eavesdropper received the packet with SINR larger than the predefined threshold $\gamma_{E}$. The secrecy outage probability, denoted as $P_{\text {out }}^{(S)}$, is then defined as the probability that secrecy outage happens during the transmission from $S$ to $D$. For a predefined upper bound $\varepsilon_{s}$ on $P_{\text {out }}^{(S)}$, we call the communication between $S$ and $D$ is secure if $P_{\text {out }}^{(S)} \leq \varepsilon_{s}$. Notice that for the transmissions from $S$ to the selected relay $R_{j^{*}}$ and from $R_{j^{*}}$ to $D$, the corresponding secrecy outage can be defined in the similar way as that of from $S$ to $D$. We use $O_{S \rightarrow R_{j^{*}}}^{(S)}$ and $O_{R_{j^{*}} \rightarrow D}^{(S)}$ to denote the events that secrecy outage from source $S$ to $R_{j^{*}}$ happens and secrecy outage from relay $R_{j^{*}}$ to $D$ happens, respectively. Again, due to the link independence assumption, we have

$$
\begin{aligned}
P_{\text {out }}^{(S)}= & P\left(O_{S \rightarrow R_{j^{*}}}^{(S)}\right)+P\left(O_{R_{j^{*} \rightarrow D}}^{(S)}\right) \\
& -P\left(O_{S \rightarrow R_{j^{*}}}^{(S)}\right) \cdot P\left(O_{R_{j^{*}} \rightarrow D}^{(S)}\right)
\end{aligned}
$$

\section{B. Analysis of Protocol 1}

In the Protocol 1 proposed in [11], the relay node with the largest value of $\min \left(\left|h_{S, R_{j}}\right|^{2},\left|h_{D, R_{j}}\right|^{2}\right), j=1,2, \cdots, n$, is selected as relay. Notice that the Protocol 1 can always guarantee the reliable transmission from source $S$ to destination $D$, this is because the parameter $\tau$ is set as $\tau=\sqrt{\frac{\log n}{8 n \gamma_{R}}}$, which ensures that the target SINR at the selected relay and destination can be achieved to decode the transmitted messages. Thus, we only need to focus the secrecy requirement $P_{\text {out }}^{(S)} \leq \varepsilon_{s}$ to determine the corresponding the number of eavesdroppers the network can tolerate here.

Theorem 1. For the network scenario illustrated in Fig 1 with equal path loss between all pairs of nodes, to guarantee the secrecy requirement $P_{\text {out }}^{(S)} \leq \varepsilon_{s}$ by applying the Protocol 1 , the number of eavesdroppers $m$ the network can tolerate should satisfy the following condition.

$$
m \leq\left(1-\sqrt{1-\varepsilon_{s}}\right) \cdot\left(1+\gamma_{E}\right)^{\sqrt{\frac{n \log n}{32 \gamma_{R}}}}
$$

Proof:

Notice that $P_{\text {out }}^{(S)}$ is determined as

$$
\begin{aligned}
P_{\text {out }}^{(S)}= & P\left(O_{S \rightarrow R_{j^{*}}}^{(S)}\right)+P\left(O_{R_{j^{*} \rightarrow D}^{(S)}}\right) \\
& -P\left(O_{S \rightarrow R_{j^{*}}}^{(S)}\right) \cdot P\left(O_{R_{j^{*} \rightarrow D}}^{(S)}\right)
\end{aligned}
$$

Since the transmission process from source $S$ to the selected relay $R_{j^{*}}$ is identical to that of from the selected relay $R_{j^{*}}$ to destination $D$, we have

$$
P\left(O_{S \rightarrow R_{j^{*}}}^{(S)}\right)=P\left(O_{R_{j^{*} \rightarrow D}}^{(S)}\right)
$$

and

$$
P_{\text {out }}^{(S)}=2 P\left(O_{S \rightarrow R_{j^{*}}}^{(S)}\right)-\left[P\left(O_{S \rightarrow R_{j^{*}}}^{(S)}\right)\right]^{2}
$$

To ensure $P_{\text {out }}^{(S)} \leq \varepsilon_{s}$, then should have

$$
P\left(O_{S \rightarrow R_{j^{*}}}^{(S)}\right) \leq 1-\sqrt{1-\varepsilon_{s}}
$$

From the reference [11], we notice that 


$$
\begin{aligned}
P\left(O_{S \rightarrow R_{j^{*}}}^{(S)}\right) & =P\left(\bigcup_{i=1}^{m}\left\{C_{S, E_{i}} \geq \gamma_{E}\right\}\right) \\
& \leq \sum_{i=1}^{m} P\left(C_{S, E_{i}} \geq \gamma_{E}\right) \\
& \leq m \cdot\left(\frac{1}{1+\gamma_{E}}\right)^{\sqrt{\frac{n \log n}{32 \gamma_{R}}}}
\end{aligned}
$$

To guarantee the secrecy requirement, we just need

$$
m \cdot\left(\frac{1}{1+\gamma_{E}}\right)^{\sqrt{\frac{n \log n}{32 \gamma_{R}}}} \leq 1-\sqrt{1-\varepsilon_{s}}
$$

and thus

$$
m \leq\left(1-\sqrt{1-\varepsilon_{s}}\right) \cdot\left(1+\gamma_{E}\right) \sqrt{\frac{n \log n}{32 \gamma_{R}}}
$$

\section{Analysis of Protocol 2}

The parameter $\tau$ involved in the Protocol 2 determines whether the relay and destination can receive the messages successfully and whether sufficient noise is generated to suppress eavesdroppers. For the analysis of the Protocol 2, we first determine the range for the parameter $\tau$ to ensure both secrecy requirement and reliability requirement, based on which we then analyze the number of eavesdroppers a network can be tolerate by applying the protocol.

Theorem 2. Consider the network scenario of Fig 1 with equal path loss between all pairs of nodes, to ensure $P_{\text {out }}^{(T)} \leq \varepsilon_{t}$ and $P_{\text {out }}^{(S)} \leq \varepsilon_{s}$ by applying the Protocol 2, the parameter $\tau$ must satisfy the following condition.

$$
\tau \in\left[-\log \left[1+\frac{\log \left(\frac{1-\sqrt{1-\varepsilon_{s}}}{m}\right)}{(n-1) \log \left(1+\gamma_{E}\right)}\right], \sqrt{\frac{-\log \left(1-\varepsilon_{t}\right)}{2 \gamma_{R}(n-1)}}\right]
$$

Proof:

Notice that in the Protocol 2, a larger value of $\tau$ indicates that more system nodes will generate noise to suppress the eavesdroppers. However, too high noise will also interrupt the legitimate transmission. Therefore, the parameter $\tau$ should be set properly to satisfy both reliability and secrecy requirements.

- Reliability Guarantee

Notice that $P_{\text {out }}^{(T)}$ is determined as

$$
\begin{aligned}
P_{\text {out }}^{(T)}= & P\left(O_{S \rightarrow R_{j^{*}}}^{(T)}\right)+P\left(O_{R_{j^{*} \rightarrow D}}^{(T)}\right) \\
& -P\left(O_{S \rightarrow R_{j^{*}}}^{(T)}\right) \cdot P\left(O_{R_{j^{*}} \rightarrow D}^{(T)}\right)
\end{aligned}
$$

Because the transmission process from source $S$ to the selected relay $R_{j^{*}}$ is identical to that of from the selected relay $R_{j *}$ to destination $D$, we have

$$
P\left(O_{S \rightarrow R_{j^{*}}}^{(T)}\right)=P\left(O_{R_{j^{*} \rightarrow D}}^{(T)}\right)
$$

and

$$
P_{\text {out }}^{(T)}=2 P\left(O_{S \rightarrow R_{j^{*}}}^{(T)}\right)-\left[P\left(O_{S \rightarrow R_{j^{*}}}^{(T)}\right)\right]^{2}
$$

To ensure $P_{\text {out }}^{(T)} \leq \varepsilon_{t}$, we need

$$
P\left(O_{S \rightarrow R_{j^{*}}}^{(T)}\right) \leq 1-\sqrt{1-\varepsilon_{t}}
$$

Based on the definition of transmission outage probability, we have

$$
\begin{aligned}
P( & \left.O_{S \rightarrow R_{j^{*}}}^{(T)}\right) \\
& =P\left(C_{S, R_{j^{*}}} \leq \gamma_{R}\right) \\
& =P\left(\frac{E_{s} \cdot\left|h_{S, R_{j^{*}}}\right|^{2}}{\sum_{R_{j} \in \mathcal{R}_{1}} E_{s} \cdot\left|h_{R_{j}, R_{j^{*}}}\right|^{2}+N_{0} / 2} \leq \gamma_{R}\right) \\
& \doteq P\left(\frac{\left|h_{S, R_{j^{*}}}\right|^{2}}{\sum_{R_{j} \in \mathcal{R}_{1}}\left|h_{R_{j}, R_{j^{*}}}\right|^{2}} \leq \gamma_{R}\right)
\end{aligned}
$$

Compared to the noise generated by multiple system nodes, the environment noise is negligible and thus is omitted here to simply the analysis. Notice that $\mathcal{R}_{1}=$ $\left\{j \neq j^{*}:\left|h_{R_{j}, R_{j^{*}}}\right|^{2}<\tau\right\}$, then

$$
\begin{aligned}
P\left(O_{S \rightarrow R_{j^{*}}}^{(T)}\right) & \leq P\left(\frac{\left|h_{S, R_{j^{*}}}\right|^{2}}{\left|\mathcal{R}_{1}\right| \tau} \leq \gamma_{R}\right) \\
& =P\left(\left|h_{S, R_{j^{*}}}\right|^{2} \leq \gamma_{R}\left|\mathcal{R}_{1}\right| \tau\right) \\
& =1-e^{-\gamma_{R}\left|\mathcal{R}_{1}\right| \tau}
\end{aligned}
$$

Since there are $n-1$ other relays except $R_{j^{*}}$, the expected number of noise-generation nodes is given by $\left|\mathcal{R}_{1}\right|=(n-1)$. $P\left(\left|h_{R_{j}, R_{j^{*}}}\right|^{2}<\tau\right)=(n-1) \cdot\left(1-e^{-\tau}\right)$. Then we have

$$
P\left(O_{S \rightarrow R_{j^{*}}}^{(T)}\right) \leq 1-e^{-\gamma_{R}(n-1)\left(1-e^{-\tau}\right) \tau}
$$

Thus, to ensure reliability requirement, we just need

$$
1-e^{-\gamma_{R}(n-1)\left(1-e^{-\tau}\right) \tau} \leq 1-\sqrt{1-\varepsilon_{t}}
$$

That is,

$$
-\gamma_{R}(n-1)\left(1-e^{-\tau}\right) \tau \geq \frac{1}{2} \log \left(1-\varepsilon_{t}\right)
$$

By using Taylor formula, we have

$$
\tau^{2} \leq \frac{-\log \left(1-\varepsilon_{t}\right)}{2 \gamma_{R}(n-1)}
$$

and thus

$$
\tau \leq \sqrt{\frac{-\log \left(1-\varepsilon_{t}\right)}{2 \gamma_{R}(n-1)}}
$$


The above result indicates that $\sqrt{\frac{-\log \left(1-\varepsilon_{t}\right)}{2 \gamma_{R}(n-1)}}$ is the maximum value the parameter $\tau$ can take to ensure the reliability requirement.

- Secrecy Guarantee

Notice that $P_{\text {out }}^{(S)}$ is given by

$$
\begin{aligned}
P_{\text {out }}^{(S)}= & P\left(O_{S \rightarrow R_{j^{*}}}^{(S)}\right)+P\left(O_{R_{j^{*} \rightarrow D}}^{(S)}\right) \\
& -P\left(O_{S \rightarrow R_{j^{*}}}^{(S)}\right) \cdot P\left(O_{R_{j^{*} \rightarrow D}}^{(S)}\right)
\end{aligned}
$$

Since the transmission process from source $S$ to the selected relay $R_{j^{*}}$ is identical to that of from the selected relay $R_{j^{*}}$ to destination $D$, then we have

$$
P\left(O_{S \rightarrow R_{j^{*}}}^{(S)}\right)=P\left(O_{R_{j^{*} \rightarrow D}}^{(S)}\right)
$$

and

$$
P_{\text {out }}^{(S)}=2 P\left(O_{S \rightarrow R_{j^{*}}}^{(S)}\right)-\left[P\left(O_{S \rightarrow R_{j^{*}}}^{(S)}\right)\right]^{2}
$$

To ensure $P_{\text {out }}^{(S)} \leq \varepsilon_{s}$, we need

$$
P\left(O_{S \rightarrow R_{j^{*}}}^{(S)}\right) \leq 1-\sqrt{1-\varepsilon_{s}}
$$

According to the definition of secrecy outage probability, we know that

$$
P\left(O_{S \rightarrow R_{j^{*}}}^{(S)}\right)=P\left(\bigcup_{i=1}^{m}\left\{C_{S, E_{i}} \geq \gamma_{E}\right\}\right)
$$

Thus, we have

$$
P\left(O_{S \rightarrow R_{j^{*}}}^{(S)}\right) \leq \sum_{i=1}^{m} P\left(C_{S, E_{i}} \geq \gamma_{E}\right)
$$

Based on the Markov inequality,

$$
\begin{aligned}
P\left(C_{S, E_{i}} \geq \gamma_{E}\right) & \\
\leq & P\left(\frac{E_{s} \cdot\left|h_{S, E_{i}}\right|^{2}}{\sum_{R_{j} \in \mathcal{R}_{1}} E_{s} \cdot\left|h_{R_{j}, E_{i}}\right|^{2}} \geq \gamma_{E}\right) \\
= & E_{\left\{h_{\left.R_{j}, E_{i}, j=0,1, \cdots, n+m p, j \neq j^{*}\right\}, \mathcal{R}_{1}}\right.} \\
& {\left[P\left(\left|h_{S, E_{i}}\right|^{2}>\gamma_{E} \cdot \sum_{R_{j} \in \mathcal{R}_{1}}\left|h_{R_{j}, E_{i}}\right|^{2}\right)\right] } \\
\leq & E_{\mathcal{R}_{1}}\left[\prod_{R_{j} \in \mathcal{R}_{1}} E_{h_{R_{j}, E_{i}}}\left[e^{\left.-\gamma_{E}\left|h_{R_{j}, E_{i}}\right|^{2}\right]}\right]\right. \\
= & E_{\mathcal{R}_{1}}\left[\left(\frac{1}{1+\gamma_{E}}\right)^{\left|\mathcal{R}_{1}\right|}\right]
\end{aligned}
$$

Therefore,
$P\left(O_{S \rightarrow R_{j^{*}}}^{(S)}\right) \leq \sum_{i=1}^{m}\left(\frac{1}{1+\gamma_{E}}\right)^{\left|\mathcal{R}_{1}\right|}=m \cdot\left(\frac{1}{1+\gamma_{E}}\right)^{\left|\mathcal{R}_{1}\right|}$

To ensure the secrecy requirement, we just need

$$
m \cdot\left(\frac{1}{1+\gamma_{E}}\right)^{\left|\mathcal{R}_{1}\right|} \leq 1-\sqrt{1-\varepsilon_{s}}
$$

or equally

$$
\begin{aligned}
& \left(\frac{1}{1+\gamma_{E}}\right)^{(n-1)\left(1-e^{-\tau}\right)} \leq \frac{1-\sqrt{1-\varepsilon_{s}}}{m} \\
& (n-1)\left(1-e^{-\tau}\right) \geq-\frac{\log \left(\frac{1-\sqrt{1-\varepsilon_{s}}}{m}\right)}{\log \left(1+\gamma_{E}\right)} \\
& e^{-\tau} \leq 1+\frac{\log \left(\frac{1-\sqrt{1-\varepsilon_{s}}}{m}\right)}{(n-1) \log \left(1+\gamma_{E}\right)}
\end{aligned}
$$

Then we have

$$
\tau \geq-\log \left[1+\frac{\log \left(\frac{1-\sqrt{1-\varepsilon_{s}}}{m}\right)}{(n-1) \log \left(1+\gamma_{E}\right)}\right]
$$

The above result shows that $-\log \left[1+\frac{\log \left(\frac{1-\sqrt{1-\varepsilon_{s}}}{m}\right)}{(n-1) \log \left(1+\gamma_{E}\right)}\right]$ is the minimum value parameter $\tau$ can take to guarantee the secrecy requirement.

Based on the results of Theorem 2, we now can establish the following theorem about the performance of Protocol 2 .

Theorem 3. Consider the network scenario of Fig 1 with equal path loss between all pairs of nodes. To guarantee $P_{\text {out }}^{(T)} \leq \varepsilon_{t}$ and $P_{\text {out }}^{(S)} \leq \varepsilon_{s}$ based on the Protocol 2, the number of eavesdroppers $m$ the network can tolerate must satisfy the following condition.

$$
m \leq\left(1-\sqrt{1-\varepsilon_{s}}\right) \cdot\left(1+\gamma_{E}\right) \sqrt{\frac{-(n-1) \log \left(1-\varepsilon_{t}\right)}{2 \gamma_{R}}}
$$

Proof:

From Theorem 2 we know that to ensure the reliability requirement, we have

$$
\tau \leq \sqrt{\frac{-\log \left(1-\varepsilon_{t}\right)}{2 \gamma_{R}(n-1)}}
$$

and

$$
(n-1)\left(1-e^{-\tau}\right) \leq \frac{-\log \left(1-\varepsilon_{t}\right)}{2 \gamma_{R} \tau}
$$

To ensure the secrecy requirement, we need 


$$
\left(\frac{1}{1+\gamma_{E}}\right)^{(n-1)\left(1-e^{-\tau}\right)} \leq \frac{1-\sqrt{1-\varepsilon_{s}}}{m}
$$

Thus,

$$
\begin{aligned}
m \leq & \frac{1-\sqrt{1-\varepsilon_{s}}}{\left(\frac{1}{1+\gamma_{E}}\right)^{(n-1)\left(1-e^{-\tau}\right)}} \\
& \leq \frac{1-\sqrt{1-\varepsilon_{s}}}{\left(\frac{1}{1+\gamma_{E}}\right)^{\frac{-\log \left(1-\varepsilon_{t}\right)}{2 \gamma_{R} \tau}}}
\end{aligned}
$$

By let $\tau$ taking its maximum value, we get the following bound

$$
m \leq \frac{1-\sqrt{1-\varepsilon_{s}}}{\left(\frac{1}{1+\gamma_{E}}\right)^{\sqrt{\frac{-(n-1) \log \left(1-\varepsilon_{t}\right)}{2 \gamma_{R}}}}}
$$

That is,

$$
m \leq\left(1-\sqrt{1-\varepsilon_{s}}\right) \cdot\left(1+\gamma_{E}\right) \sqrt{\frac{-(n-1) \log \left(1-\varepsilon_{t}\right)}{2 \gamma_{R}}}
$$

\section{Discussion}

The two protocols considered in this paper have their own advantages and disadvantages and thus are suitable for different network scenarios. For the protocol 1 proposed in [11], it can achieve a better performance in terms of the number of eavesdroppers can be tolerated. However, such protocol always tend to select the optimal node with the best links to both source and destination as the relay, so it involves a complex relay selection process, and more importantly, it results in an unbalanced load and energy consumption distribution among systems nodes. Thus, such protocol is suitable for small scale wireless network with sufficient energy supply rather than large and energy-limited wireless networks (like wireless sensor networks). Regarding the Protocol 2, although it can tolerate less number eavesdroppers in comparison with the Protocol 1 , it involves a very simple random relay selection process to achieve a very good load and energy consumption distribution among system nodes. Thus, this protocol is more suitable for large scale wireless network environment with stringent energy consumption constraint.

\section{CONCLUSION}

This paper explores reliable and secure information transmission through multiple cooperative systems nodes in two-hop relay wireless network with passive eavesdroppers of unknown channels and locations, for which two transmission protocols are considered. For each protocol, theoretical analysis has been provided to show the number of eavesdroppers the network can tolerate subject to constraints on transmission outage probability and secrecy outage probability. These two protocols, each has different performance in terms of eavesdropper tolerance, load and energy consumption distribution among nodes, and also relay selection complexity, are suitable for different network scenarios depending on network scale and also energy consumption constraint there.

\section{REFERENCES}

[1] Narayanan, Sathya, Two-hop forwarding in wireless networks, dissertation for the degree of Doctor of philosophy, Polytechnic University, 2006

[2] J. Talbot and D. Welsh, Complexity and Crytography : An Introduction, Cambridge, 2006.

[3] K. Morrison, and D. GoeckelPower allocation to noise-generating nodes for cooperative secrecy in the wireless environment. In the Forty Fifth Asilomar Conference on Signals, Systems and Computers (ASILOMAR), 275-279, 2011.

[4] S. Goel, and R. Negi, Guaranteeing secrecy using artificial noise. IEEE transactions on wireless communications, 7(6):2180-2189, 2008.

[5] L. Lai and H. El Gamal, The relay-eavesdropper channel: Cooperation for secrecy, IEEE Trans. Inf. Theory, vol. 54, no. 9, pp. 4005 - 4019 , Sept. 2008.

[6] M. Yuksel and E. Erkip, Secure communication with a relay helping the wiretapper, in Proc. 2007 IEEE Information Theory Workshop, Lake Tahoe, CA, Sept. 2007.

[7] R. Negi and S. Goelm, Secret communication using artificial noise, in Proc. IEEE Vehicular Tech. Conf, vol. 3, Dallas TX, pp. 1906-1910, Sept. 2005.

[8] S. Vasudevan, S. Adams, D. Geockel, Z. Ding, D. Towsley, and K. Leung, Multi-user diversity for secrecy in wireless networks. In Information Theorem and Applications Workshop, 2009.

[9] X. He and A. Yener, Two-hop secure communication using an untrusted relay: A case for cooperative jamming, in Proc. 2008 IEEE Global Telecommunications Conference, New Orleans, LA, Nov. - Dec. 2008.

[10] L. Dong, Z. Han, A. Petropulu, and H. V. Poor, Improving wireless physical layer security via cooperating relays, IEEE Trans. Sig. Proc., vol. 58, no. 3, pp. 1875-1888, Mar. 2010.

[11] D. Goeckel, S. Vasudevan, D. Towsley, S. Adams, Z. Ding, and K. Leung, Artificial noise generation from cooperative relays for everlasting secrecy in two-hop wireless networks, IEEE Journal on Selected Areas in Communications, 29(10):2067-2076, 2011. 